\title{
Technology and Topology Optimization for Multizonal Transmission Systems
}

\author{
Hakan Ergun Graduate Student Member, IEEE, Barry Rawn Member, IEEE, \\ Ronnie Belmans Fellow, IEEE and Dirk Van Hertem Senior Member, IEEE
}

\begin{abstract}
This paper presents a method to optimize equipment investments in multizonal transmission systems, considering spatial properties of the areas of focus. Together with a probabilistic technique for assessing nodal injection capability, the method in the paper completes the methodology of a long term transmission system planning tool. Transmission topology, line route and technology are optimized through iterative application of linear integer programming and Dijkstra's shortest path algorithm. By optimizing the transmission route, the spatial properties of the area of focus are taken into account, which in turn can significantly influence the installation costs of transmission equipment. The optimization method considers both AC and DC technology and takes the $\mathrm{N}-1$ security criterion into account.
\end{abstract}

Index Terms-Transmission System Expansion Planning, Transmission System Optimization, Optimal Cable Routing, HVDC, Transmission System Investments

\section{INTRODUCTION}

Transmission system investment optimization is a difficult task due to the non-linear, non-convex and mixed-integer nature of the optimization problem. The market liberalization and integration of energy from renewable energy sources introduce additional difficulties. In the liberalized market, the optimal dispatch of generators in different price zones with different rules must be taken into account in order to maximize social welfare for a transnational transmission network. When renewable power flows begin to dominate a network, new combinations of generation and load become possible and occur in a wider range. Yet in classical transmission system investment optimization, injections of generators have usually been assumed as being fixed [i]].

In literature, a large number of transmission system optimization methodologies exist. Mathematical optimization methods include linear programming [I]-[6], quadratic programming , non-linear programming [ [ $]$ ], integer programming and mixed-integer programming [8]-[15] and their combinations. For high dimensional non-convex problems these methods become computationally expensive and have been enhanced by heuristic methods. The most popular include sensitivity analysis [16]-[22], genetic algorithms [23]-[29], simulated annealing [30], [31], tabu search [32]-[34] and particle swarm optimization [35]. Also game theory based methods can be considered as heuristic methods, as the game follows some predefined rules [36]. Most successful optimization achieves scalability by decomposing the problem at hand into different parts [4].

H. Ergun, B. Rawn, R. Belmans and D. Van Hertem are with the Department of Electrical Engineering (ESAT/ELECTA), KU Leuven, Kasteelpark Arenberg 10, 3001 Heverlee, Belgium, http://www.esat.kuleuven.be/electa.

Contact: hakan.ergun@esat.kuleuven.be
A key short coming of available planning methodologies is that the probabilistic nature of renewable generation and demand is not considered. For large transnational investments robustness is essential. If the transmission system planning is scenario based, both over investment or under investment could occur. This is because scenario studies may easily overestimate future generation capacities. At the same time, they could miss extreme cases of flow that require special reinforcement.

Another important issue which is neglected by most planning methodologies is the possibility of using HVDC technology. The optimal topology and rating of a transmission system may change in the case of HVDC technology. Especially for high power ratings, long distances and submarine applications, HVDC is an attractive possible solution. Additionally, VSC HVDC offers flexibility due to the ability of controlling power flow and voltage in the existing grid. Therefore the possibility of grid expansion using HVDC should be considered. Current methodologies also tend to neglect cabling (installation of overhead lines vs. underground cabling) and routing of the transmission system although they influence transmission system costs and therefore the optimal solution. The decision of building overhead lines or underground cables as well using HVAC or HVDC technology is influenced by different geographic, environmental and sociological conditions which should be taken into account. The topology, rating, technology, cabling and routing of transmission systems are interdependent and should be optimized together as such.

This paper proposes a methodology to optimize routing, technology, ratings and cabling of transnational transmission systems by iteratively using MILP optimization and Dijkstra's shortest path algorithm. The necessary input for the optimization is determined using probabilistic techniques taking the merit order of generation units into account as described in [37]. The N-1 criterion is respected during the design of the transnational transmission system.

\section{PRoposed PLANNING METHOdology AND OPTIMIZATION METHOD}

The proposed optimization methodology is part of a long term planning tool to stepwise optimize transmission system investments for large scale renewable integration as depicted in fig. W. It is divided in three major parts for each time step of the planning horizon. First, the necessary optimal transmission capability between two price zones is calculated. The optimal interconnection power can be approximated using a market model considering the merit order of generators in both zones and linear cost functions for the necessary transmission expan- 


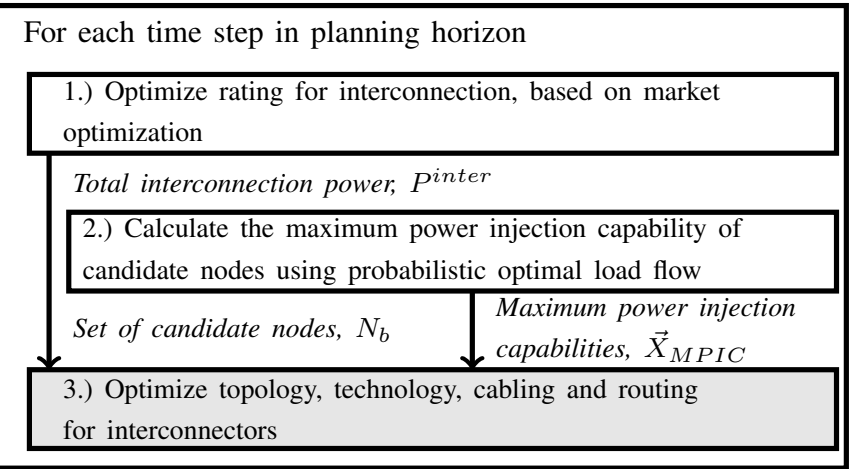

Fig. 1: Overview of the long term transmission planning method for large scale renewable integration. The focus of this paper in on the optimization method of Step 3.

sion. In the second step, the maximum power injection capability of candidate nodes for the interconnectors are calculated, which serve as input for the topology optimization. During the calculation, the uncertainty in generation and demand and their effects on the merit order of committed generators is taken into account. A methodology to calculate the maximum power injection capability of the under laying grid is provided in [37].

The focus of this paper is on the third step of the long term planning tool. The aim of the optimization method in this step is to find the best transmission topology, technology, cabling and routing between two zones for a given total interconnection power, based on the power injection capability of the chosen candidate nodes. The optimal routing, cabling option and technology of a transmission link will be different depending on the link's rated power. At the same time, when these elements are optimized, their cost will reduce, possibly changing the importance of that link in the optimization of topology.

If all the optimal solutions per path and power rating are known, the optimal topology can be determined using linear integer programming. It is however a time intensive prospect to calculate the optimal route, technology and cabling option for every possible power rating and every possible path. The method explained in this paper separates the coupled problem into two dependent sub-problems: the selection of topology and rating of an electrical network, and the discovery of a least cost path which combines the factors of routing, technology and cabling. The two problems are iteratively solved in turn. Topology and rating are first optimized using mixed-integer linear programming (MILP), but based only on fixed average costs per branch. Only the branches chosen in the first stage for a given iteration are optimally routed using Dijkstra's shortest path algorithm [38]. Sequentially, the average costs used in the first stage are updated based on the results of the optimal routing algorithm for the next iteration. This way, the computation time can be decreased significantly. The case study of this paper shows that global solutions are still approached.

Fig. $\square$ depicts the optimization method. To solve the optimization problem, the maximum power injection capabilities of a selected set of buses have to be known. This input is

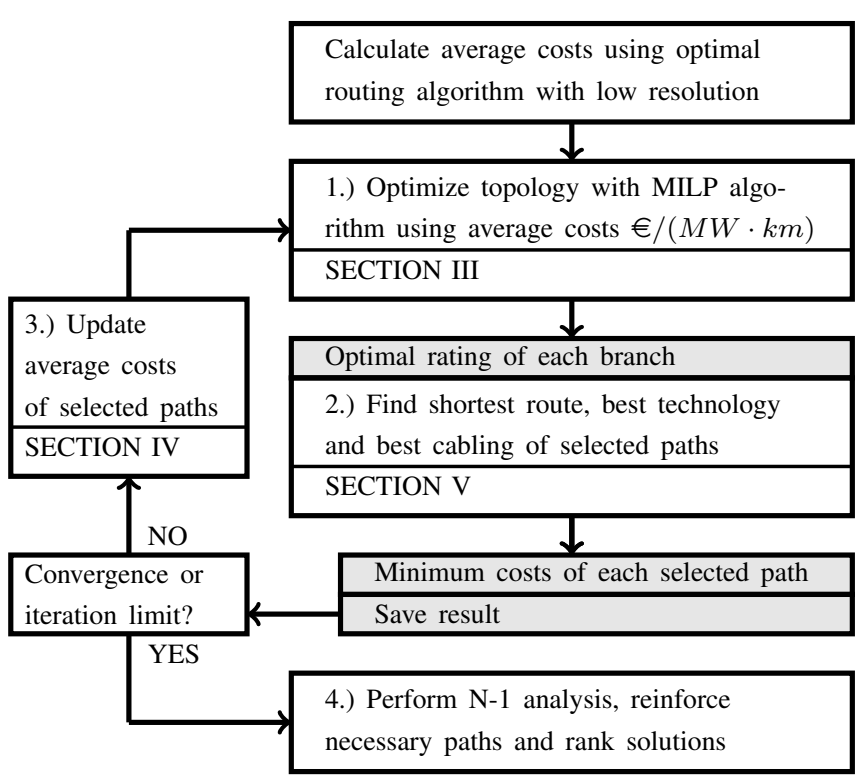

Fig. 2: Iterative optimization of transmission topology, technology, cabling and routing

determined in the second step of the afore mentioned long term planning method (fig. 四).

Initially, average costs for each possible branch are determined, which are used as input for the MILP optimization. To save computation time, these costs are determined using the optimal routing algorithm with a low spatial resolution. The average costs have the dimension $€ / M W$. A detailed explanation of the cost calculation is given in Section $\mathbb{Z}$.

In the first step, the transmission system topology is optimized using MILP. In this step the rating of each branch is optimized, using the average costs and the distance between buses. The objective function and constraints of the MILP optimization are described in Section W.

In the second step, the optimal routing, technology and cabling of each selected path is determined. To do so, spatial information of the investigated area is required. Based on the spatial information, different sub areas with different spatial weights for each technology and cabling option are defined. These spatial weights are translated into costs to be used in the optimal routing algorithm, which is described in Section $\nabla$. The output of the optimal routing algorithm are the minimum costs, the optimal route, the technology and cabling option for each selected transmission path and power rating.

In the third step, the average costs of selected paths are updated using the output of the optimal routing algorithm. The obtained topology is saved and the routine is repeated until the objective function value of the MILP algorithm and the optimal routing algorithm converge or a certain number of iterations is reached. After the iterative optimization, circuit additions are made to the obtained transmission solutions to fulfill the N-1 security criterion.

\section{MILP OPTIMIZATION OF TOPOLOGY AND RATING}

Using a desired interconnection power $P^{i n t e r}$ as input, the goal of the MILP optimization is to find the optimal rating of 
branches in a given set of possible branches. Possible ratings are restricted to discrete values given by the $1 \times N_{k}$ vector

$$
\mathbf{k}=\left[k_{1}, k_{2}, \ldots, k_{N_{k}}\right]
$$

where $N_{k}$ is the number of possible ratings allowed. The set of possible branches consists of all possible branches between candidate nodes. If there are $N_{n}$ candidate nodes, $N_{b}=N_{n} ! /\left(2 \cdot\left(N_{n}-2\right) !\right)$ possible branches exist.

The search space consists of $N_{s}=2 \cdot N_{b} \cdot N_{k}$ variables. The interconnector can be comprised of multiple tie-lines and branches, and the final optimal configuration may require flow in different directions. Each branch may affect maximum possible injections of other nodes in a manner unsymmetrical with direction. To account for asymmetry and flow cancellation, two decision matrices of size $N_{b} \times N_{k}$ are used in the formulation to indicate a given topology and capacity design, with the definition for positive paths being:

$$
U^{+}=\left\{\begin{array}{cc}
u_{i, j}=1 & \text { if branch } i \text { with rating } j \text { selected } \\
0 & \text { otherwise }
\end{array}\right.
$$

and the definition for $U^{-}$being similar. Each branch can have at most one capacity specified and a path cannot be selected to be both forward and backward (i.e. the elementwise product and sum or Frobenius norm of the decision matrices $\sum_{i} \sum_{j} U_{i j}^{+} U_{i j}^{-}=0$ ). Some branches are also tielines as marked by the indicator $N_{b} \times 1$ vector

$$
\mathbf{t}=\left\{\begin{array}{cc}
1 & \text { if branch } i \text { is a tie line } \\
0 & \text { otherwise }
\end{array} .\right.
$$

The actual capacities selected are a $N_{b} \times 1$ vector

$$
K=\left(U^{+}-U^{-}\right) \cdot \mathbf{k}
$$

where sign of $K$ indicates intended direction of flow to achieve $P^{\text {inter. }}$.

The topology optimization problem is formulated as the minimization of a total capacity cost $C_{c a p}$ while meeting or exceeding the desired interconnection power and not exceeding maximum injection capabilities:

$$
\begin{aligned}
\min _{\substack{\forall U^{+}, U^{-} \\
\in B^{N} \times N_{k}}} C_{c a p} & =\left(U^{+}+U^{-}\right):(\overrightarrow{1} \otimes \mathbf{k}) \cdot \tilde{C}: L \\
& =\sum_{i} \sum_{j}\left(U_{i j}^{+}+U_{i j}^{-}\right) \cdot K_{i} \cdot \tilde{C}_{i j} L_{i j} \\
\text { s.t. } & \\
P^{\text {inter }} & \leq\left(U^{+}+U^{-}\right): \mathbf{t} \otimes \mathbf{k} \\
P_{M P I C} & \geq \Delta_{M P I C} \cdot\left(U^{+}+U^{-}\right) \cdot \mathbf{k} \\
\bar{K}, \forall i \in\left[1, N_{b}\right] & \geq K_{i}
\end{aligned}
$$

where $\tilde{C}_{i j}$ and $L_{i j}$ denote an average cost and length respectively for every possible branch $i$ and rating $j, P_{M P I C}$ is a column vector with the nodal maximum power injection capability and $\triangle_{M P I C}$ is a matrix defining the change in maximum power injection capabilities due to flows on selected candidate branches. The methodology to calculate $P_{M P I C}$ and $\Delta_{M P I C}$ are provided in appendix $\mathbf{\Delta} . \bar{K}$ is the maximum allowable capacity of a single line dictated by reliability concerns. As indicated in the expansion (5)), the ":" operator in (5) and (G) is the sum over an elementwise product or Frobenius product, while "." denotes a regular matrix multiplication or inner product and $\otimes$ the outer product.

Constraint (6) states that the sum of power ratings of interconnecting branches has to be more than or equal to the total interconnection power. $P^{\text {inter }}$ is the total interconnection power provided by the long term planning tool. Constraint (प) describes Kirchhoff's current law and states that the maximum power injection capability of connected nodes may not be exceeded. $P_{M P I C}$ is a $N_{n} \times 1$ vector containing maximum power injection capabilities of candidate buses. If there is a power injection or withdrawal in one node, the maximum power injection capabilities of other nodes will change, which is expressed by $\Delta_{M P I C}$. The dependence of $P_{M P I C}$ on grid design should be robustly determined according to the the probabilistic nature of generation and demand as well as the merit order of existing generators into account using the methodology provided in [37].

Constraint (8) states that the power rating of a selected branch $i$ must not exceed a defined maximum rating $\overline{\mathbf{k}}$, as determined by the technology associated with that branch in the current iteration.

The optimization problem is implemented in MATLAB. The SCIP solver is used to solve the MILP problem [39].

\section{Calculation AND update of AVerage cost MATRIX $\tilde{C}$}

The average transmission system costs in $€ / M W$ are inversely proportional to the rating of the transmission system. A given technology will achieve a desired link capacity by using an integer number of lines. This introduces a discontinuity in the dependence of cost on link capacities, as shown in fig. Ba. If different technologies and cabling options are considered, the number of discontinuities found in the neighborhood of a given link capacity value can increase. Instead, either technology may be cheapest depending on the nominal capacity range. A local minimum always comes next to such discontinuities, and this complicates the optimization of the topology. Fig. Ba also demonstrates that there can be multiple breakeven values to guide a choice of technology 1 or 2 . Fig. Bb shows that for different power ratings of the interconnector, different technologies might provide the cheapest solution.

To provide average costs for the first iteration, the costs per $M W$ for each possible path at the maximum power rating of one system are calculated. The calculation is performed for four different possibilities, namely AC underground cables (AC UGC), AC overhead lines (AC OHL), DC underground cables (DC UGC) and DC overhead lines (DC OHL), using the optimal routing algorithm with a low spatial resolution. In the same way, the costs at the maximum power rating of a single path, $\bar{K}$ (e.g. on one tower of overhead transmission lines) are calculated. The costs at power ratings other than the afore mentioned ratings are obtained using a linear approximation of the calculated costs (fig. taa).

After each iteration, the costs of selected paths and power ratings are updated using the costs calculated with the optimal 


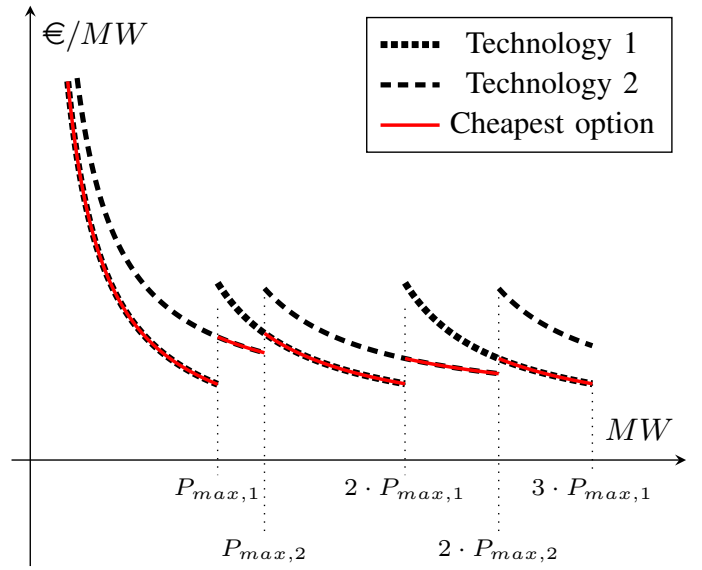

(a) Average transmission system costs as a function of the power rating fro two different technologies.

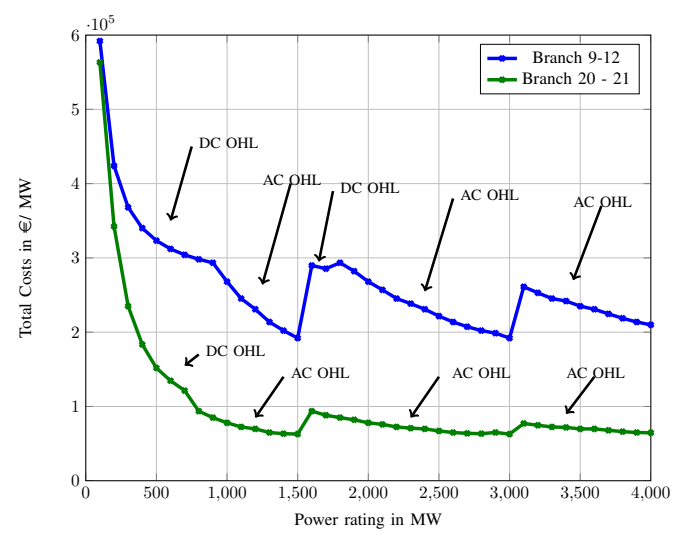

(b) Costs per MW for two possible branches of the case study depicted in fig. 6 . The cheapest technology option for each power range is indicated.

Fig. 3: Average transmission system costs in $€ / M W$ as a function of rated power in MW

routing algorithm with higher resolution. The costs at all other power ratings are recalculated using linear interpolation and extrapolation of the updated costs (Fig. 4b). The MILP might get stuck in a local optimum if no penalty functions are used while updating the average costs. Therefore, if the costs after the route optimization are lower than the assumed average costs, the average costs of not selected branches are decreased. In contrary, if the costs after route optimization are higher than the assumed average costs, the costs for the selected branch are increased for all power ratings. This way, new branches and power ratings become more attractive during the MILP optimization.

\section{Optimal Routing Formulation and Solution FROM COST GRAPH}

In transmission investment planning, it is not sufficient to consider only overhead lines for this purpose. Due to increased public opposition, permissions to build overhead lines (OHL) are hard to obtain [40]. When it comes to AC UGC, the possible transmission distance is limited due to the high cable capacitance and the resulting charging currents. Therefore DC transmission has to be considered as well.

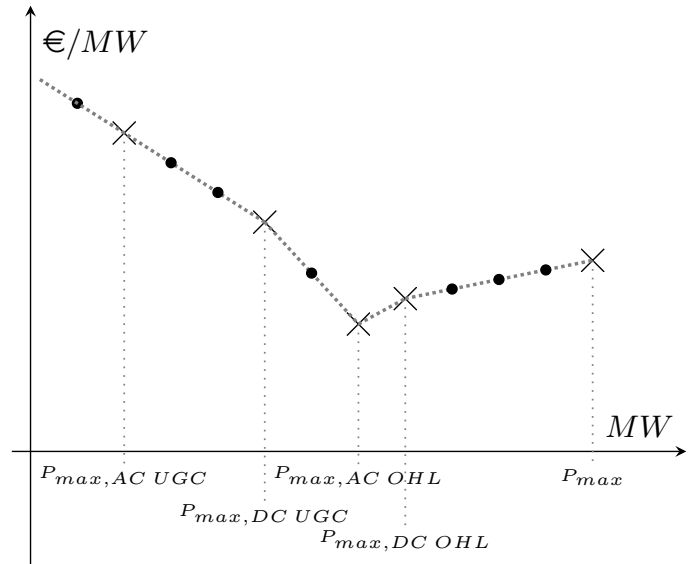

(a) Calculation of average costs for initial MILP

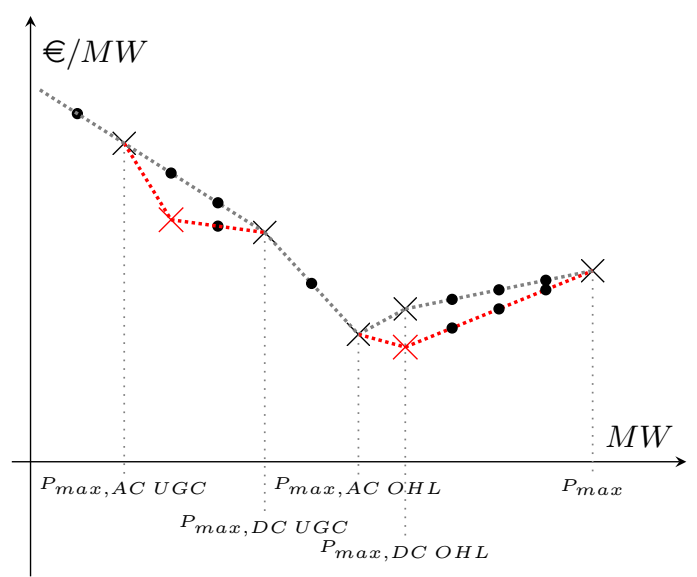

(b) Update of average costs after each iteration . Red line indicates the costs used in the next iteration

Fig. 4: Calculation of initial and updated average costs for the MILP optimization. Crosses indicate costs calculated by the optimal routing algorithm. Dots indicate extrapolated costs.

The route and transmission technology are interdependent. Additionally, the installation costs for different technologies depend on the type of soil, land acquisition costs and other factors. It is therefore important to optimize the cabling option (OHL vs. UGC), technology (HVAC vs. HVDC) and the transmission route at the same time.

The installation costs for transmission equipment depend on spatial properties of the area of focus. Therefore, a map of the area is discretized to a grid of spatial points $p_{i}=\left[x_{i}, y_{i}\right]$ of size $N_{x} \times N_{y}$. To be able to optimize the technology together with the cabling option, the four technologies of AC OHL, AC UGC, DC OHL and DC UGC must also be represented, as well as the effect of creating hybrid options such as $\mathrm{AC}$ with DC and OHL with UGC.

The optimal routing and technology problem is formulated around a weighted graph $\mathcal{G}=(\mathcal{V}, \mathcal{E})$ having four technology layers and weighted edges signifying costs. For every spatial point $p$, there are four associated vertices $\mathcal{V}$ representing possible technologies, as outlined in figs. 5a and 5b. A full set of edges $\mathcal{E}$ between vertices associated with adjacent spatial points correspond to cables or lines of a given technology. There are also edges between the vertices associated 
with different technologies at the same spatial point $p$ that correspond to mechanical or electrical conversions necessary to join dissimilar technologies. This means that for a graph with $N_{x} \cdot N_{y}$ nodes, $\left(10 \cdot N_{x} \cdot N_{y}-3 \cdot\left(N_{x}+N_{y}\right)+2\right)$ edges exist.

The graph cost function $\mathcal{W}\left(p_{i}, \mathcal{E}_{i}\right)$ can take on a different positive value for every edge $j$ and represents the cost associated with progressing spatially or switching between technologies:

$\mathcal{W}\left(p_{i}, \mathcal{E}_{j}\right)=\left(c^{i n v}\left(\mathcal{E}_{j}\right)+c^{i n s t}\left(\mathcal{E}_{j}\right) \cdot w\left(p_{i}\right)\right) d\left(\mathcal{E}_{j}\right)+w^{\text {switch }}\left(\mathcal{E}_{j}\right)$

where $c^{i n v}\left(\mathcal{E}_{j}\right)$ is a technology-dependent investment cost and $c^{i n s t}\left(p_{i}, \mathcal{E}_{j}\right)$ corresponds to the installation cost dependent on both location and technology with $w\left(p_{i}\right)$ a spatial weighting factor. $d\left(\mathcal{E}_{j}\right)$ is the spatial distance associated with the edge, and $w^{\text {switch }}\left(\mathcal{E}_{j}\right)$ is a technology conversion cost. The cost factors are also dependent on the optimal capacity $K$ determined by the other sub-problem, but this is omitted as the quantity is fixed for a given iteration.

In $(\mathbb{Q}), c^{i n v}\left(\mathcal{E}_{j}\right)$ and $c^{i n s t}\left(\mathcal{E}_{j}\right)$ are given in $€ / k m$ for a defined reference area. The spatial weight $w\left(p_{i}\right)$ defines the relative difference of installation costs in a certain area compared to the reference area. In case of offshore cabling a different $c^{i n v}\left(\mathcal{E}_{j}\right)$ is used to take price differences between onshore and offshore cables into account. In the same manner, different prices are used for offshore and onshore HVDC converters to take the significant offshore platform costs into account. To take the additional costs for the switch in technology into account, the weighting factor $w^{\text {switch }}\left(\mathcal{E}_{j}\right)$ is assigned to each edge of the graph. Fig. $\$$ shows how the technology switch is realized. The four technology maps (fig. 5a) are connected at each vertex $x 1 / y 1$ to each other. In case of a switch from $\mathrm{AC}$ to $\mathrm{DC}$ or $\mathrm{DC}$ to $\mathrm{AC}$, the edge is weighted with the cost of an HVDC converter. In case of a switch within the same technology to a different cabling option, the weight is determined using the necessary number of cable systems and conductors per cable system (fig. 5b). If there is no switch in technology or cabling option, $w_{i}^{\text {switch }}$ is zero.

To calculate the investment costs for the four transmission options, the number of conductors and their cross sections have to be known. The minimum number of conductors and their cross section are calculated using the rated power of one transmission path (delivered by the MILP algorithm) and the rated voltage. $\mathrm{AC}$ and $\mathrm{DC}$ technology are assigned $400 \mathrm{kV}$ and $\pm 320 \mathrm{kV}$ respectively.

High voltage AC UGC have a high capacitance resulting in high charging currents. This imposes a maximum distance on a cable without compensation. It is therefore assumed that the cable has to be compensated after each cable section of the length $\bar{d}_{a c-u g c}$ (10).

$$
\bar{d}_{a c, u g c}=\frac{\sqrt{3} I_{N}}{\sqrt{2} \cdot \omega \cdot C \cdot U_{N}}
$$

In (10), $I_{N}$ is the nominal current of one conductor, $C$ the capacitance of the conductor per $k m$ and $U_{N}$ the rated voltage of the conductor. Using the charging current, the rating and cost of the necessary compensation equipment is determined and added to the investment costs of AC cables.

To realize longer sections of $\mathrm{AC}$ offshore cables in one path, the cable route must pass an through onshore area for compensation. Therefore, a distinction in the Dijkstra algorithm is made between offshore and onshore edges of the AC UGC graph. Every time when an edge located offshore is chosen, the offshore length of the selected path is compared to the maximum AC offshore length $\bar{d}_{a c, u g c}$. If the maximum offshore length of one path is reached, the weights of the next candidate offshore AC edges are multiplied with a large number, so that they are not chosen by the algorithm. After a switch from offshore $\mathrm{AC}$ to onshore $\mathrm{AC}$ or to HVDC technology, the offshore length of the path is set to zero again to enable other possible segments with maximum AC offshore length.

The algorithm to optimize the routing of a transmission path together with the used technology and the cabling option is based on Dijkstra's shortest path algorithm [38]. Dijkstra's algorithm finds the shortest path between two defined vertices in a weighted graph. If the weights assigned to the edges of the graph are costs per $\mathrm{km}$ of transmission line, Dijkstra's algorithm delivers the minimum transmission equipment cost between two vertices (एI). As all four technology layers are interconnected, the Dijkstra algorithm delivers the minimum transmission system cost

$$
\min _{S} C_{\text {equip }}=\sum_{\mathcal{E} \in \mathcal{S}} \mathcal{W}(\mathcal{E})
$$

where $S$ is a sequence of edges corresponding to the lowest cost or "shortest" path. The edges between the four technology graphs have per definition a length of zero.

\section{CASE STUDY}

The developed optimization method is applied to a case study with 10 candidate nodes as shown in Fig. 6. The maximum power injection capabilities of the candidate buses are calculated for the IEEE 24 bus test system [41] with modified generation, load and branch flow limits, according [37]. The calculated maximum power injection capabilities of

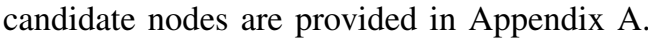

In the case study, the test system is divided in two zones. Zone 1 consists of buses 1 to 12 and bus 24, and zone 2 consists of buses 13 to 23 [4]]. In both zones, 5 buses are chosen as candidate buses which can serve as connection points for interconnectors between the two zones. Fig. 6 shows the division of the area of focus in several sub areas influencing the installation costs of transmission equipment. The spatial weights reflecting installation costs of the four different technology options are provided in Table II. These values are chosen arbitrarily and do not necessarily reflect real installation and land acquisition cost ratios. Costs, reflecting soft constraints such as electromagnetic fields, visual and environmental impact can also be included in these spatial weights.

A number of case studies are analyzed to verify that the proposed methodology converges towards the global optimal solution. Therefore, the total interconnection power, $P^{\text {inter }}$ 

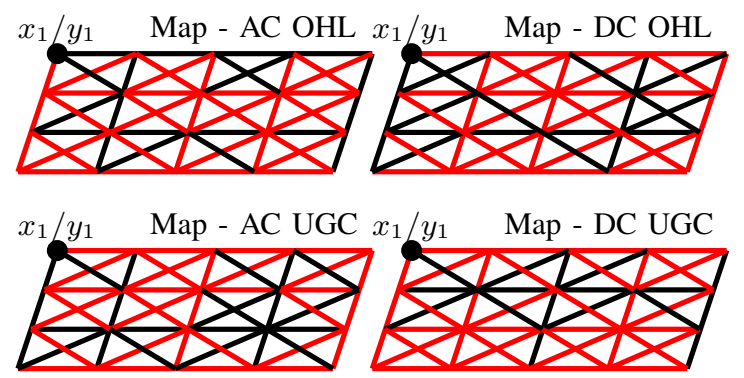

(a) Illustration of the weighted graphs for the 4 technologies, different colors indicate different weights

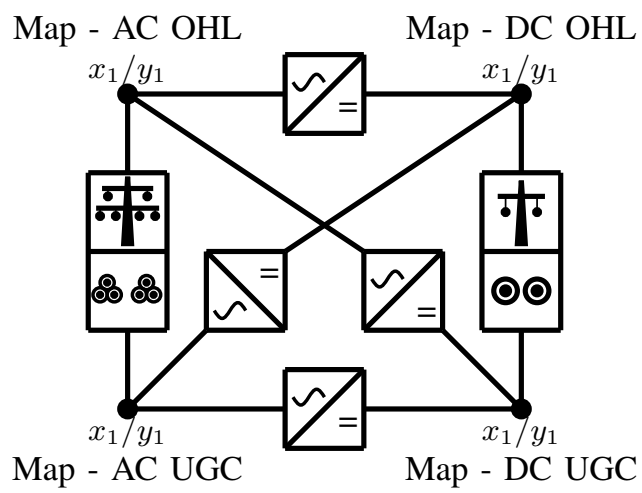

(b) Connection of the four weighted graphs at each edge to enable the technology switch

Fig. 5: Creation of a weighted directed graph containing four technologies as input for the Dijkstra algorithm

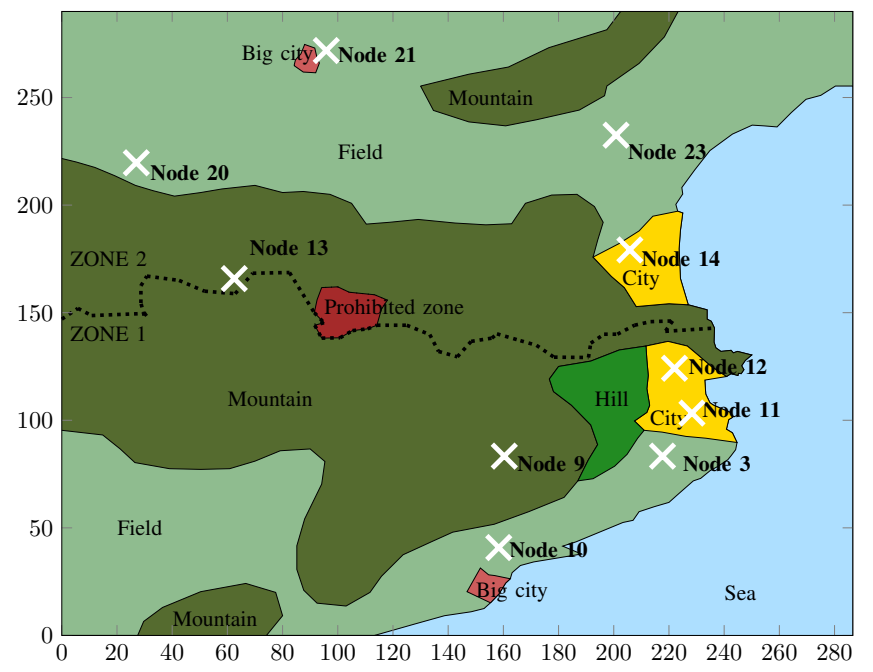

Fig. 6: Spatial map of area of focus showing candidate nodes in both zones The case study resembles the eastern border between France and Spain, although no attempt is made to obtain actual correct results.

and the maximum power per path, $\bar{K}$ are varied. During the calculations, the spatial map as shown in Fig. 6 is used. As possible power ratings of transmission paths, multiples of $100 \mathrm{MW}$ are considered. The total cost

$$
C_{\text {tot }}=\sum_{N_{b}} C_{\text {equipment }}
$$

is the final outcome of the optimization methodology. Table $\mathbb{I}$ compares the computation time as well as the cost difference
TABLE I

SPATIAL WEIGHTS OF THE SUB AREAS FOR THE DIFFERENT TECHNOLOGY OPTIONS

\begin{tabular}{lcccc} 
Area & AC OHL & DC OHL & AC UGC & DC UGC \\
\hline Field & 1 & 1 & 1 & 1 \\
Hill & 10 & 8 & 2 & 1.5 \\
Mountain & 15 & 12 & 6 & 4.5 \\
Sea & 40 & 40 & 1 & 0.75 \\
City & 20 & 16 & 3 & 2.5 \\
Big city & 40 & 40 & 3 & 2.5 \\
Prohibited area & 40 & 40 & 40 & 40 \\
\hline
\end{tabular}

between the solution obtained with the proposed method and global optimum. The global optimum is found using a brute force approach. Therefore, the minimum costs for each possible transmission path and power rating are determined using the optimal routing algorithm as shown in section $\nabla$. Using these minimal costs as input, the global optimum is calculated using the MILP algorithm as described in section IIII.

Table II shows that near optimal solutions can be found in a fraction of the computation time. In case of higher resolutions, more computation time is saved with the proposed method. The reason is that the computation time for the optimal routing algorithm increases exponentially with the resolution, whereas the computation time for the MILP algorithm is approximately constant as the number of optimization variables does not change.

Figure $\square$ and Table $\mathbb{\text { II }}$ show how the resolution of spatial map discretization can affect the final optimal grid topology.

TABLE II

COMPARISON OF PROPOSED METHOD (PRO) TO BRUTE FORCE CALCULATION (BFC), EXCLUDING N-1

\begin{tabular}{ccccccc}
$\begin{array}{c}P^{\text {inter }} \\
(\mathrm{MW})\end{array}$ & $\bar{K}$ & $\begin{array}{c}\text { Resolution } \\
(\mathrm{MW})\end{array}$ & $\begin{array}{c}C_{\text {tot }} \\
(\mathrm{km})\end{array}$ & $\begin{array}{c}\Delta C_{\text {tot }} \\
(\mathrm{M} €)\end{array}$ & \multicolumn{2}{c}{ Time $(\mathrm{min})$} \\
\hline 7000 & 2000 & 4 & 1983.7 & 0 & 58 & $>2880$ \\
& & 8 & 1931.4 & 0.06 & 10 & $>120$ \\
\hline 7000 & \multirow{2}{*}{3000} & 4 & 1983.7 & 0.24 & 56 & $>4320$ \\
& & 8 & 1916 & 0.59 & 16 & $>180$ \\
\hline 6000 & 2000 & 4 & 1680.5 & 0 & 60 & $>2880$ \\
& & 8 & 1628.7 & 0.043 & 12 & $>120$ \\
\hline 6000 & \multirow{2}{*}{3000} & 4 & 1680.5 & 1.94 & 116 & $>4320$ \\
& & 8 & 1621.9 & 0.466 & 23 & $>180$ \\
\hline
\end{tabular}

To obtain the global optimum for the N-1 secure design, security constraints have to be added to both the MILP and the optimal routing algorithm which would increase the computation time drastically. As shown in fig. ฉ, the proposed method saves the optimal solution of each iteration. The optimal solution among all iterations is obtained, after making circuit additions ensuring $\mathrm{N}-1$ security for each obtained grid topology. Table III compares the total costs after necessary circuit additions. In column 4 , the total costs are shown which include additions to the global optimum found with brute force approach in the $\mathrm{N}$ case. Column 5 shows the optimal $\mathrm{N}-1$ solution obtained with the proposed method. It can be observed that suboptimal solutions in the $\mathrm{N}$ case can deliver better solutions in the $\mathrm{N}-1$ case. Fig. 8 shows such a case. In the $\mathrm{N}$ case, the costs of the depicted solution add up to $2135.5 \mathrm{M} €$ and are $10.06 \%$ higher than the global optimum, whereas in the $\mathrm{N}-1$ case, the depicted case achieves cost 


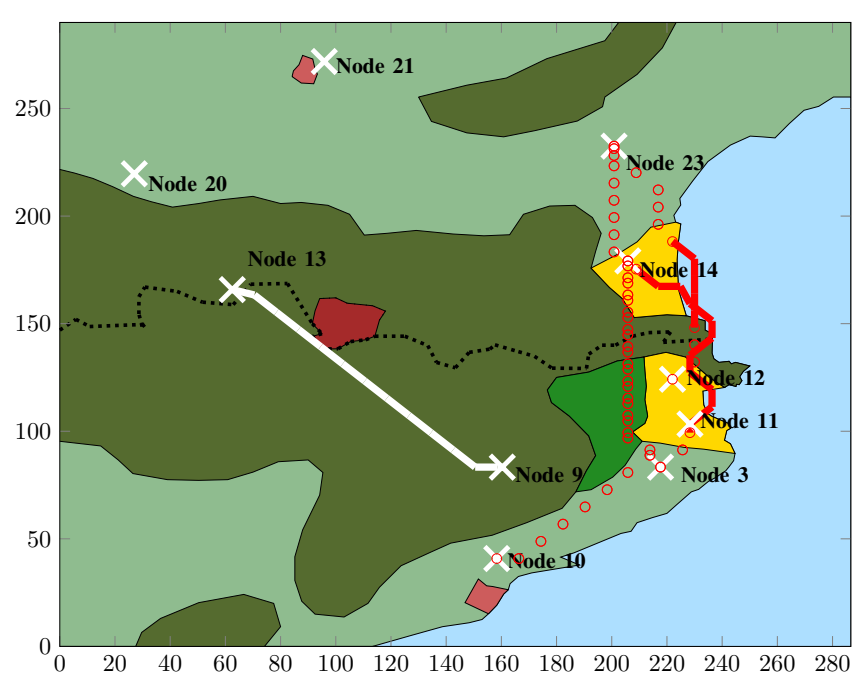

(a) Optimal topology calculated with a resolution of $8 \mathrm{~km}$ excluding $\mathrm{N}-1$

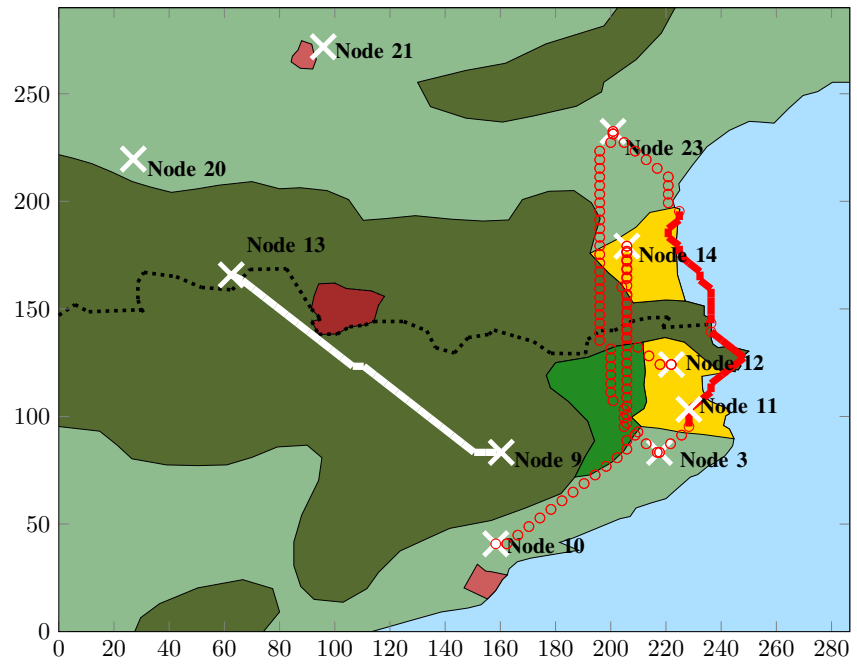

(b) Optimal topology calculated with a resolution of $4 \mathrm{~km}$ excluding $\mathrm{N}-1$

Fig. 7: Comparison of different resolutions. Red color indicates HVAC connections. White color indicates HVDC connections. Solid lines indicate underground cables. Circles indicate overhead lines. $P^{\text {inter }}=7000 \mathrm{MW}$, $\overline{\mathbf{K}}=2000 M W$

savings of $9.58 \%$ compared to the global optimum of the $\mathrm{N}$ case with necessary circuit additions.

\section{TABLE III}

COSTS INCLUDING CIRCUIT ADDITIONS FOR N-1 SECURITY

\begin{tabular}{cccccc}
$\begin{array}{c}P_{\text {inter }} \\
(\mathrm{MW})\end{array}$ & $\bar{K}$ & $\begin{array}{c}\text { Resolution } \\
(\mathrm{MW})\end{array}$ & \multicolumn{2}{c}{$C_{\text {tot }}$} & $\Delta C_{\text {tot }}$ \\
\hline 7000 & 2000 & 4 & 3545.8 & 3206.1 & -9.58 \\
& & 8 & 3232.9 & 3092.8 & -4.33 \\
\hline 7000 & \multirow{2}{*}{3000} & 4 & 3367.4 & 3223.1 & -4.29 \\
& & 8 & 2982.2 & 2610.9 & -12.45 \\
\hline 6000 & 2000 & 4 & 2859.1 & 2583.2 & -9.65 \\
& & 8 & 2590.7 & 2366.6 & -8.65 \\
\hline 6000 & \multirow{2}{*}{3000} & 4 & 2964.8 & 2586.8 & -12.75 \\
& & 8 & 2560 & 2036.9 & -20.43 \\
\hline
\end{tabular}

The necessary additional investments to make the grid $\mathrm{N}$ 1 secure add up to $1560 \mathrm{M} €$ for the depicted solution in

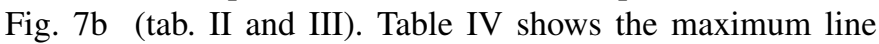
overload $\left(P_{o l, \max }\right)$, the probability of failure $\left(p_{f}\right)$, the mean

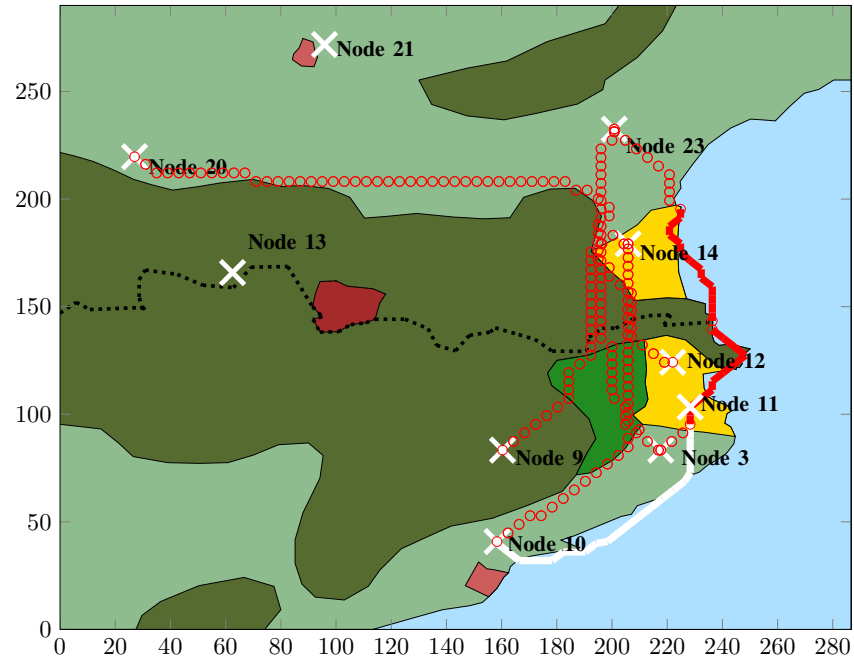

Fig. 8: Optimal topology calculated with a resolution of $4 \mathrm{~km}$ including N-1 for $P^{\text {inter }}=7000 M W, \overline{\mathbf{K}}=2000 M W$

time to repair ( $m t t r)$ and the calculated costs for the not served energy $\left(C_{\text {ens }}\right)$ assuming a lifetime of 25 years for the solution depicted in Fig. $\square$. As costs for not served energy $100 € / \mathrm{MWh}$ are used. Table $\mathbb{} \mathbb{V}$ shows that the costs for not served energy add up to $789.9 \mathrm{M} €$ over a lifetime of 25 years. This means that the costs for not served energy should be higher than $197.8 € / M W h$ to justify the investments into the $\mathrm{N}-1$ secure scheme.

TABLE IV

\section{COST OF NOT SERVED ENERGY}

\begin{tabular}{ccccr}
$\begin{array}{c}\text { Branch } \\
\text { outage } \\
\text { from-to }\end{array}$ & $\begin{array}{c}P_{\text {ol,max }} \\
\text { MW }\end{array}$ & $\begin{array}{c}p_{f} \\
1 /(\mathrm{km} \cdot \text { year }) \\
\text { offsh/onsh/ohl }\end{array}$ & $\begin{array}{c}\text { mttr } \\
\text { days } \\
\text { offsh/onsh/ohl }\end{array}$ & $\begin{array}{r}C_{\text {ens }} \\
\text { M€ }\end{array}$ \\
\hline $10-14$ & 1500 & 0.00353 & 0.125 & 8.65 \\
$9-13$ & 1500 & $21 \cdot 10^{-4}$ & 10 & 248.89 \\
$3-11$ & 1100 & $21 \cdot 10^{-4} / 0.00353$ & $10 / 0.125$ & 30.89 \\
$11-23$ & 900 & $21 \cdot 10^{-4} / 0.00353$ & $30 / 10 / 0.125$ & 498.03 \\
$12-14$ & 2000 & 0.00353 & 0.125 & 3.43 \\
\hline
\end{tabular}

\section{CONCLUSION}

This paper shows a methodology to optimize multizonal transmission system investments. The advantage of the proposed methodology is that the spatial properties of the area of focus are taken into account. The best transmission route and technology are determined depending on the area specific installation costs of transmission system equipment.

The performance of the optimal routing algorithm only depends on the chosen resolution to discretize the given area of focus, whereas the performance of the MILP algorithm depends on the number of possible nodes and power ratings. That means in presence of an efficient MILP solver, this methodology can be applied to larger systems as well.

The optimization of the transmission route together with the transmission technology allows to include area specific costs for soft constraints like electromagnetic emissions, visual and environmental impacts of the transmission system, which are strong decision criteria for realization of transmission projects. 
With the developed methodology, if known, area specific costs for the soft constraints can be assigned to the spatial weights and considered during the optimization.

The paper illustrates that the methodology is able to find near optimum solutions in a fraction of the computation time required to find the global optimum. The methodology includes the $\mathrm{N}-1$ security criterion by making circuit additions after determining the optimal solution for the $\mathrm{N}$ case.

From the point of view of not supplied energy, the feasibility of designing the system $\mathrm{N}-1$ secure heavily depends on the costs associated to the not supplied energy. If these costs are known, investments in additional circuits can be optimized.

\section{ACKNOWLEDGEMENT}

This work is supported by ABB, Elia and Vattenfall in the framework of the industrial research project "Reaching for a Pan-European Grid: How to Make the Transition? Transmission System Investment Planning”.

\section{APPENDIX A \\ Calculation of $P_{M P I C}$ And $\Delta_{M P I C}$}

This section is based on [37] and briefly explains the calculation of the $P_{M P I C}$ vector and the $\Delta_{M P I C}$ matrix. $P_{M P I C}$ illustrates the maximum power injection capabilities whereas $\Delta_{M P I C}$ expresses how the maximum power injection capability of certain nodes in the power system change due to injections in other nodes. During the calculation of $P_{M P I C}$ and $\Delta_{M P I C}$, the probabilistic nature of generators and loads as well as the cost of generation is taken into account using a probabilistic optimal load flow approach.

The maximum power injection capability (MPIC) is calculated using the objective function in ([1]) where $n g$ is the number of generators, $f_{p}^{i}\left(p_{g}^{i}\right)$ the generation costs of generator $i$ as a function of its power injection, $p_{g}^{x}$ the power injection of the investigated node $x$ and $K$ a scaling factor. The optimization constraints are the flow limits of transmission lines, voltage magnitudes, voltage angle limits, active and reactive power limits of generators, the Kirchhoff equations and the system load-generation balance.

$$
\min \sum_{i=1}^{n g} f_{p}^{i}\left(p_{g}^{i}\right)-K p_{g}^{x}
$$

Using probabilistic load and generation profiles and varying node $x$ between all nodes in the system, a range of possible maximum power injections can be obtained for each node as shown in fig. $Q$. The figure shows the mean value of the maximum power injection capability $\bar{P}$, its standard deviation $\sigma$, the most critical branch $C B$ and how often $C B$ reaches its limits when the maximum injection occurs $(L L F)$.

Let us consider a node $x^{1}$. To see the change in the MPIC of node $x^{1}$ depending on the injection in another node $x^{2}$, a generator is added in node $x^{2}$. The generator has negative production costs so it will be selected by the OPF algorithm described above. The power rating of the generator is varied among all possible power ratings used in section $\mathrm{Wl}$. This way, the change in the MPIC of node $x^{1}$ depending on the injection in node $x^{2}$ can be calculated $\left(\Delta_{1,2,1}\right)$. In case of absorptions,

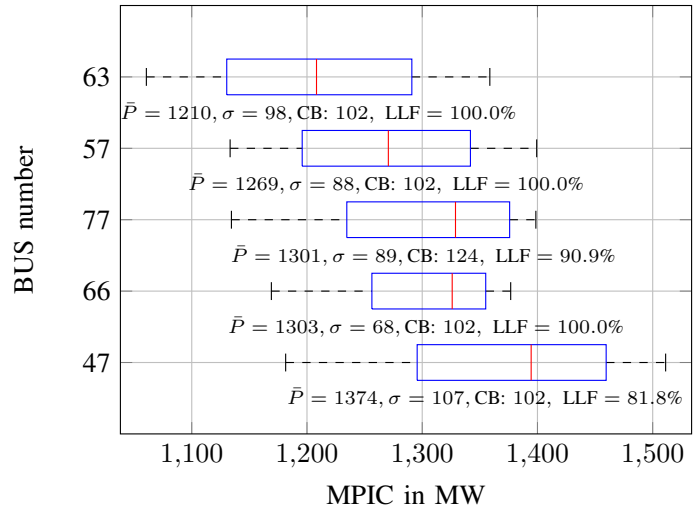

Fig. 9: Maximum power injection capabilities of selected nodes in the IEEE 118 bus system [37]

the additional generators are replaced by loads. (III) shows the structure of $\Delta_{M P I C}$. The first index indicates the affected node, the second index indicates the effecting nodes and the third index indicates the power rating.

$\Delta_{M P I C}=\left[\begin{array}{ccccccc}\Delta_{1,1,1} & \Delta_{1,2,1} & \ldots & \Delta_{1, N, 1} & \ldots & \Delta_{1, N, N} & \\ \Delta_{2,1,1} & \Delta_{2,2,1} & \ldots & \Delta_{2, N, 1} & \ldots & \Delta_{2, N, N} & \\ \vdots & \vdots & \vdots & \vdots & \vdots & \vdots & \vdots \\ \Delta_{N, 1,1} & \Delta_{N, 2,1} & \ldots & \Delta_{N, N, 1} & \ldots & \Delta_{N, N, N} & ]_{(14)}\end{array}\right.$

Tab. $\nabla$ shows the $P_{M P I C}$ vector such as used in the case study.

TABLE V

$P_{M P I C}$ USED FOR THE CASE STUDY

\begin{tabular}{lcc} 
Bus & Injection Cap. in MW & Withdrawal Cap. in MW \\
\hline 3 & 3029 & 1324 \\
9 & 3719 & 1668 \\
10 & 4117 & 1481 \\
11 & 1787 & 1670 \\
12 & 1147 & 1713 \\
13 & 1990 & 6199 \\
14 & 2969 & 2279 \\
20 & 1793 & 5955 \\
21 & 5180 & 6315 \\
23 & 2701 & 6399 \\
\hline
\end{tabular}

\section{REFERENCES}

[1] L. Garver, "Transmission Network Estimation Using Linear Programming," Power Apparatus and Systems, IEEE Transactions on, vol. PAS89, no. 7, pp. $1688-1697$, sept. 1970.

[2] R. Villasana, L. L. Garver, and S. J. Salon, "Transmission Network Planning Using Linear Programming," Power Engineering Review, IEEE, vol. PER-5, no. 2, pp. 36 -37, feb. 1985.

[3] V. Levi and M. Calovic, "Linear-programming-based decomposition method for optimal planning of transmission network investments," Generation, Transmission and Distribution, IEE Proceedings C, vol. 140, no. 6 , pp. $516-522$, nov 1993.

[4] _ "A new decomposition based method for optimal expansion planning of large transmission networks," Power Systems, IEEE Transactions on, vol. 6, no. 3, pp. $937-943$, aug 1991.

[5] R. Chanda and P. Bhattacharjee, "Application of computer software in transmission expansion planning using variable load structure," Electric Power Systems Research, vol. 31, no. 1, pp. 13 - 20, 1994.

[6] K. Kim, Y. Park, and K. Lee, "Optimal long term transmission expansion planning based on maximum principle," Power Systems, IEEE Transactions on, vol. 3, no. 4, pp. $1494-1501$, nov 1988.

[7] H. Youssef and R. Hackam, "New transmission planning model," Power Systems, IEEE Transactions on, vol. 4, no. 1, pp. 9 -18, feb 1989.

[8] V. A. Levi, "A new mixed-integer methodology for optimal transmission expansion planning," Electric Power Systems Research, vol. 32, no. 3, pp. $227-238,1995$. 
[9] L. Bahiense, G. Oliveira, M. Pereira, and S. Granville, "A mixed integer disjunctive model for transmission network expansion," Power Systems, IEEE Transactions on, vol. 16, no. 3, pp. 560 -565, aug 2001.

[10] S. Haffner, A. Monticelli, A. Garcia, J. Mantovani, and R. Romero, "Branch and bound algorithm for transmission system expansion planning using a transportation model," Generation, Transmission and Distribution, IEE Proceedings-, vol. 147, no. 3, pp. 149 -156, may 2000.

[11] A. Seifu, S. Salon, and G. List, "Optimization of transmission line planning including security constraints," Power Systems, IEEE Transactions on, vol. 4, no. 4, pp. $1507-1513$, nov 1989

[12] J. dos Santos, A., P. Franca, and A. Said, "An optimization model for long-range transmission expansion planning," Power Systems, IEEE Transactions on, vol. 4, no. 1, pp. 94 -101, feb 1989.

[13] A. Sharifnia and H. Aashtiani, "Transmission Network Planning: A Method for Synthesis of Minimum-Cost Secure Networks," Power Apparatus and Systems, IEEE Transactions on, vol. PAS-104, no. 8 , pp. $2025-2034$, aug. 1985.

[14] A. Meliopoulos, R. Webb, R. Bennon, and J. Juves, "Optimal Long Range Transmission Planning with AC Load Flow," Power Apparatus and Systems, IEEE Transactions on, vol. PAS-101, no. 10, pp. 4156 -4163 , oct. 1982.

[15] S. Lee, K. Hicks, and E. Hnyilicza, "Transmission Expansion by Branchand-Bound Integer Programming with Optimal Cost - Capacity Curves,' Power Apparatus and Systems, IEEE Transactions on, vol. PAS-93, no. 5 pp. $1390-1400$, sept. 1974.

[16] M. Pereira and L. Pinto, "Application Of Sensitivity Analysis Of Load Supplying Capability To Interactive Transmission Expansion Planning,' Power Apparatus and Systems, IEEE Transactions on, vol. PAS-104 no. 2, pp. $381-389$, feb. 1985 .

[17] A. Ekwue and B. Cory, "Transmission System Expansion Planning by Interactive Methods," Power Apparatus and Systems, IEEE Transactions on, vol. PAS-103, no. 7, pp. 1583 -1591, july 1984

[18] A. Ekwue, "Investigations of the transmission system expansion problem," International Journal of Electrical Power; Energy Systems, vol. 6 , no. 3, pp. 139 - 142, 1984.

[19] A. Monticelli, A. Santos, M. Pereira, S. Cunha, B. Parker, and J. Praca "Interactive Transmission Network Planning Using a Least-Effort Criterion," Power Apparatus and Systems, IEEE Transactions on, vol. PAS101 , no. 10 , pp. $3919-3925$, oct. 1982.

[20] R. Bennon, J. Juves, and A. Meliopoulos, "Use of Sensitivity Analysis in Automated Transmission Planning," Power Apparatus and Systems, IEEE Transactions on, vol. PAS-101, no. 1, pp. 53 -59, jan. 1982.

[21] C. Dechamps and E. Jamoulle, "Interactive computer program for planning the expansion of meshed transmission networks," International Journal of Electrical Power; Energy Systems, vol. 2, no. 2, pp. 103 $108,1980$.

[22] C. Serna, J. Duran, and A. Camargo, "A Model for Expansion Planning of Transmission Systems A Practical Application Example," Power Apparatus and Systems, IEEE Transactions on, vol. PAS-97, no. 2, pp $610-615$, march 1978.

[23] E. Da Silva, H. Gil, and J. Areiza, "Transmission Network Expansion Planning under an Improved Genetic Algorithm," in Power Industry Computer Applications, 1999. PICA '99. Proceedings of the 21st 1999 IEEE International Conference, jul 1999, pp. 315 -321.

[24] _ "Transmission Network Expansion Planning under an Improved Genetic Algorithm," Power Systems, IEEE Transactions on, vol. 15 no. 3, pp. $1168-1174$, aug 2000

[25] K. Yoshimoto, K. Yasuda, and R. Yokoyama, "Transmission expansion planning using neuro-computing hybridized with genetic algorithm," in Evolutionary Computation, 1995., IEEE International Conference on vol. 1, nov-1 dec 1995, p. 126.

[26] R. Gallego, A. Monticelli, and R. Romero, "Comparative studies on nonconvex optimization methods for transmission network expansion planning," Power Systems, IEEE Transactions on, vol. 13, no. 3, pp. $822-828$, aug 1998.

[27] — , "Transmission system expansion planning by an extended genetic algorithm," Generation, Transmission and Distribution, IEE Proceedings-, vol. 145, no. 3, pp. 329 -335, may 1998

[28] A. Escobar, R. A. Gallego, R. L. Romero, and S. A. de Oliveira, "Hybrid model analysis and constructive algorithms in the performance of a genetic algorithm for the planning of an electric power transmission system," Proc. 17th Int. Conf. CARS and FOF, 2001.

[29] H. Gil and E. Da Silva, "A reliable approach for solving the transmission network expansion planning problem using genetic algorithms," Electric Power Systems Research, vol. 58, no. 1, pp. 45 - 51, 2001.

[30] R. Romero, R. Gallego, and A. Monticelli, "Transmission system ex pansion planning by simulated annealing," in Power Industry Computer
Application Conference, 1995. Conference Proceedings., 1995 IEEE, may 1995 , pp. $278-283$.

[31] R. Gallego, A. Alves, A. Monticelli, and R. Romero, "Parallel simulated annealing applied to long term transmission network expansion planning," Power Systems, IEEE Transactions on, vol. 12, no. 1, pp $181-188$, feb 1997.

[32] R. Gallego, R. Romero, and A. Monticelli, "Tabu search algorithm for network synthesis," Power Systems, IEEE Transactions on, vol. 15, no. 2, pp. $490-495$, may 2000

[33] E. Da Silva, J. Ortiz, G. De Oliveira, and S. Binato, "Transmission network expansion planning under a Tabu Search approach," Power Systems, IEEE Transactions on, vol. 16, no. 1, pp. 62 -68, feb 2001.

[34] F. Wen and C. Chang, "Transmission network optimal planning using the tabu search method," Electric Power Systems Research, vol. 42, no. 2, pp. $153-163,1997$.

[35] P. Sensarma, M. Rahmani, and A. Carvalho, "A comprehensive method for optimal expansion planning using particle swarm optimization," in Power Engineering Society Winter Meeting, 2002. IEEE, vol. 2, 2002, pp. $1317-1322$ vol.2.

[36] A. Orths and Z. Styczynski, "Game theoretical approach to power network planning," in Power Tech Proceedings, 2001 IEEE Porto, vol. 1, 2001, p. 6 pp. vol.1.

[37] H. Ergun, D. Van Hertem, and R. Belmans, "Identification of Power Injection Capabilities for Transmission System Investment Optimization,' 10th IEEE PowerTech Conference, June 2013, Grenoble-France.

[38] E. Dijkstra, "A Note on Two Problems in Connexion with Graphs," Numerische Mathematik, vol. 1, no. 1, pp. 269-271, 1959.

[39] T. Achterberg, "Constraint integer programming," Ph.D. dissertation, 2009. [Online]. Available: scip.zib.de/

[40] S. P. Vajjhala and P. S. Fischbeck, "Quantifying siting difficulty: A case study of us transmission line siting," Energy Policy, vol. 35, no. 1, pp. $650-671,2007$

[41] C. Grigg, P. Wong, P. Albrecht, R. Allan, M. Bhavaraju, R. Billinton, Q. Chen, C. Fong, S. Haddad, S. Kuruganty, W. Li, R. Mukerji, D. Patton, N. Rau, D. Reppen, A. Schneider, M. Shahidehpour, and C. Singh, "The IEEE Reliability Test System-1996. A report prepared by the Reliability Test System Task Force of the Application of Probability Methods Subcommittee," Power Systems, IEEE Transactions on, vol. 14, no. 3, pp. 1010-1020, 1999.

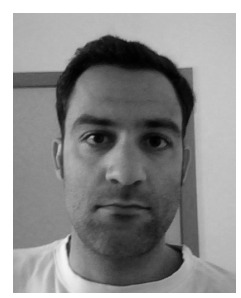

Hakan Ergun (S'10) received his degree in electrical engineering (Dipl.-Ing.) at the Technical University Graz, Austria in 2009. Since 2010 is he research assistant at KU Leuven and pursuing a $\mathrm{PhD}$ degree. His filed is long term transmission system planning and development for large scale renewable integration. He is an active member of IEEE and the chair of the PES Student Branch Chapter Leuven.

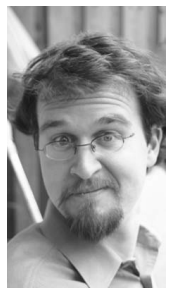

Barry Rawn (M'10) received the $\mathrm{PhD}$ degree in electrical engineering in 2010 from the University of Toronto, where he also received the BASc and MASc degrees in Engineering Science and Electrical Engineering. His research interests include nonlinear dynamics and sustainable energy infrastructure. He is currently a postdoctoral researcher in the ELECTA group at the KU Leuven, Belgium. 


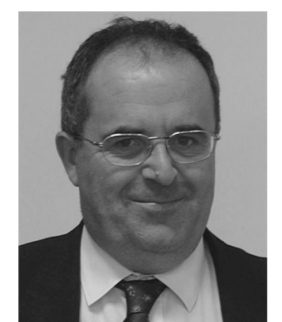

Ronnie Belmans (S'77-M'84-SM'89-F'05) received the M.Sc. degree in electrical engineering in 1979 and the Ph.D. degree in 1984, both from the University of Leuven (KU Leuven), Belgium, the Special Doctorate in 1989 and the Habilitierung in 1993, both from the RWTH, Aachen, Germany. Currently, he is a full professor with the KU Leuven, teaching electric power and energy systems. His research interests are within the framework of grids, smart grids, the deployment of all the new grid technologies and their integration with new resources and the demand in electricity. He is also Guest Professor at Imperial College of Science, Medicine and Technology, London, UK. Dr. Belmans is a Fellow of the IEEE and the IET. He is also the CEO of EnergyVille, the Executive Director of the Global Smart Grid Federation and Honorary Chairman of the board of Elia, the Belgian transmission grid operator.

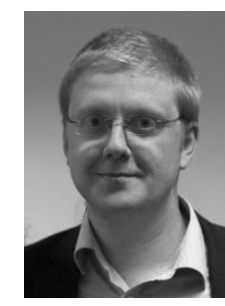

Dirk Van Hertem (S'02, SM'09) was born in 1979, in Neerpelt, Belgium. He graduated as a M.Eng. in 2001 from the KHK, Geel, Belgium and as a M.Sc. in Electrical Engineering from the KU Leuven, Belgium in 2003. In 2009, he has obtained his $\mathrm{PhD}$ also from the KU Leuven. In 2010, Dirk Van Hertem was a member of EPS group at the Royal Institute of Technology, in Stockholm, Sweden where he was the program manager for controllable power systems for the $\mathrm{EKC}^{2}$ competence center at KTH. Since spring 2011 he is back at the University of Leuven where he is an assistant professor in the ELECTA group. His special fields of interest are power system operation and control in systems with FACTS and HVDC and building the transmission system of the future, including offshore grids and the supergrid concept. He is an active member of both IEEE (PES and IAS) and Cigré. 